\title{
Do Statins Affect Thyroid Volume and Nodule Size in Patients with Hyperlipidemia in a Region with Mild-to-Moderate lodine Deficiency? A Prospective Study
}

\author{
Canan Demir $^{\mathrm{a}}$ Cuneyd Anil ${ }^{\mathrm{a}}$ Yusuf Bozkus $^{\mathrm{a}}$ Umut Mousa $^{\mathrm{a}}$ Altug Kut $^{\mathrm{b}}$ \\ Asli Nar ${ }^{a}$ Neslihan B. Tutuncu ${ }^{a}$ \\ a Department of Endocrinology and Metabolism, Baskent University School of Medicine, Ankara, Turkey; \\ ${ }^{b}$ Department Family Medicine, Baskent University School of Medicine, Ankara, Turkey
}

\section{Significance of the Study}

- This study compared the thyroid function and morphology of hyperlipidemic patients who received atorvastatin or rosuvastatin with those of a control group of non-statin-treated hyperlipidemic patients. There was an association between rosuvastatin treatment and reduction in thyroid volume and maximum nodule diameter. This could be a new treatment alternative for benign and malignant proliferative thyroid diseases.

\section{Keywords}

Hyperlipidemia $\cdot$ HMG-CoA reductase inhibitor $\cdot$ Statins . Atorvastatin · Rosuvastatin · Thyroid volume $\cdot$ Thyroid nodule

\begin{abstract}
Objective: The objective of this study was to assess the antiproliferative pleiotropic effects of statins on thyroid function, volume, and nodularity. Subjects and Methods: One hundred and six hyperlipidemic patients were included in this prospective study. The 69 patients in the statin groups received atorvastatin ( 16 received $10 \mathrm{mg}$ and 18 received 20 $\mathrm{mg}$ ) or rosuvastatin (20 received $10 \mathrm{mg}$ and 15 received 20 $\mathrm{mg}$ ). The 37 patients in the control group, assessed as not requiring drugs, made only lifestyle changes. Upon admis-
\end{abstract}

\begin{tabular}{ll}
\hline KARGER & $\begin{array}{l}\text { ( ) 2018 The Author(s) } \\
\text { Published by S. Karger AG, Basel Openger }\end{array}$ \\
E-Mail karger@karger.com & $\begin{array}{l}\text { This is an Open Access article licensed under the Creative Commons } \\
\text { Attribution-NonCommercial-4.0 International License (CC BY-NC) } \\
\text { (http://www.karger.com/Services/OpenAccessLicense), applicable to } \\
\text { the online version of the article only. Usage and distribution for } \\
\text { commercial purposes requires written permission. }\end{array}$
\end{tabular}

sion and after 6 months, all patients were evaluated by ultrasonography as well as for lipid variables (total cholesterol, high- and low-density lipoprotein cholesterol, and triglycerides) and thyroid function and structure. Results: After 6 months, no differences in thyroid function, thyroid volume, the number of thyroid nodules, or nodule size were observed in the statin and control groups. In a subgroup analysis, total thyroid volume had decreased more in patients receiving $20 \mathrm{mg}$ of rosuvastatin than that in the control group $(p<0.05)$. Maximum nodule size had decreased more in those receiving $10 \mathrm{mg}$ of rosuvastatin $(p<0.05)$. Conclusions: Our results suggest an association between rosuvastatin treatment and smaller thyroid volume and maximum nodule diameter; this could be attributable to the antiproliferative effects of statin therapy on the thyroid.

(c) 2018 The Author(s)

Published by S. Karger AG, Basel

Cuneyd Anil

Department of Endocrinology and Metabolism, Baskent University School of Medicine Fevzi Cakmak Cad., 10. Sok., No. 45, Bahcelievler

TR-06490 Ankara (Turkey)

E-Mail cuneydanil@yahoo.com 


\section{Introduction}

Statins inhibit 3-hydroxy-3-methylglutaryl-coenzyme A (HMG-CoA) reductase enzyme, the rate-limiting step in cholesterol synthesis, and thus prevent cholesterol synthesis through mevalonate [1]. The effects of statins apart from lipid reduction are known as pleiotropic effects and are believed to be attributable to the inhibition of mevalonate and isoprenoid compound synthesis via the inhibition of HMG-CoA reductase [2,3]. The mevalonate pathway provides the bioactive molecules essential for numerous cellular processes. The by-products of the mevalonate pathway, farnesyl pyrophosphate and geranylgeranyl pyrophosphate, enable $G$ proteins to be transported from within cells to the cell membranes and activated by posttranslational modification.

The resultant activated proteins of the Ras super-family participate in controlling processes such as cell proliferation and differentiation, and the regulation of the actin cytoskeleton, membrane traffic, and nuclear transport. Many studies have shown that the mechanism responsible for statin pleiotropy is the inhibition of the Ras superfamily guanosine triphosphate (GTP)ases, which act as regulators in guanosine diphosphate/GTP transformation, and whose membrane localizations and functions depend on posttranslational protein isoprenylation [4$6]$. When the pleiotropic effects of statins were first recognized, various studies investigated their antitumor, antiproliferative, and apoptotic effects [7-10].

Abnormal epithelial cell proliferation, which is common in thyroid tissue, may present as nodular or nonnodular thyroid growth or neoplasia [11]. However, little is known about the pleiotropic effects of statins on thyroid cells. A possible thyroid-statin association was first reported in 1999, when Vitale et al. [12] showed in vitro that statins induce apoptosis in "multiplying thyroid cells" independently of p53, through protein synthesis and a mechanism based on caspase enzymes. Rat thyroid cells with Ras-transformation were found to be more sensitive to HMG-CoA reductase inhibitors than normal cells. Ras mutation activation and other oncogenes are common in thyroid tumors; ras mutations may also be present in nodular hyperplasia of the thyroid $[13,14]$. In a 2006 study performed on rats, Laezza et al. [15] showed that HMG-CoA reductase inhibitors inhibit propylthiouracil-induced goiter with p21 ras-MAPK (mitogen-activated protein kinase) pathway modulation. Thus, statins may offer a new treatment alternative for benign and malignant proliferative thyroid diseases. Only 2 retrospective and no prospective clinical studies on the pleiotropic
Table 1. Relevant characteristics of the study population

\begin{tabular}{lccc}
\hline & $\begin{array}{l}\text { Control group } \\
(n=37)\end{array}$ & $\begin{array}{l}\text { Statins group } \\
(n=69)\end{array}$ & $p$ \\
\hline Age, years & $51.68 \pm 15$ & $55.7 \pm 7.8$ & 0.142 \\
Sex & & & \\
$\quad$ Female & $24(64.9)$ & $38(55.1)$ & 0.409 \\
$\quad$ Male & $13(35.1)$ & $31(44.9)$ & \\
Weight, kg & $76.8 \pm 15.3$ & $77.5 \pm 12.9$ & 0.810 \\
BMI & $27.8 \pm 4.2$ & $28.5 \pm 4.2$ & 0.414 \\
Smoking status & $7(18.9)$ & $19(27.5)$ & 0.554 \\
Comorbidity & $4(10.8)$ & $38(55.1)$ & $<0.001^{*}$ \\
$\quad$ DM & $11(29.7)$ & $38(55.1)$ & $0.015^{*}$ \\
$\quad$ HT & $4(10.8)$ & $8(11.6)$ & 0.589 \\
$\quad$ CHD & & &
\end{tabular}

Values are expressed as means \pm SD or $n(\%)$. DM, diabetes mellitus; HT, hypertension; CHD, coronary heart disease.

$* p<0.05$.

effects of statins on the thyroid have been published in English $[16,17]$. In this study, the objective was to prospectively compare the change in thyroid function and morphology of hyperlipidemic patients receiving atorvastatin or rosuvastatin with those of a control group of non-statin-treated hyperlipidemic patients in a region with mild-to-moderate iodine deficiency. Thyroid volume and morphology are affected by iodine intake, with the incidence of goiter and nodules being higher in iodine-deficient regions $[18,19]$.

\section{Subjects and Methods}

\section{Subjects}

This prospective, controlled study took place from August 2012 to March 2014 at the Department of Endocrinology and Metabolism, Baskent University School of Medicine, Ankara, Turkey. The study was approved by the local institutional review board and written informed consent was obtained from all participants.

One hundred and seventeen patients who presented to our hospital and were diagnosed with hypercholesterolemia were enrolled. The National Cholesterol Education Program Adult Treatment Panel (NCEP ATP) III guidelines [20] on drug treatment indications were used to plan the hyperlipidemia treatment. After assessment for the risk of coronary heart disease, 80 patients with indications for drug treatment and no exclusion criteria according to NCEP ATP III guidelines (despite a 4-week first-stage diet) were assigned to various "statin" groups. Exclusion criteria included previous statin treatment, a known current or previous thyroid disease, current levothyroxine replacement therapy, current or previous use of antithyroid drugs, radioactive iodine therapy, and current or previous use of drugs that may affect thyroid functions. Eleven patients were excluded from the drug intervention groups
Demir/Anil/Bozkus/Mousa/Kut/Nar/ Tutuncu 
Table 2. Mean lipid concentrations before and after intervention in all groups

\begin{tabular}{|c|c|c|c|c|c|c|c|c|c|c|}
\hline & \multicolumn{2}{|c|}{ Control $(n=37)$} & \multicolumn{2}{|c|}{$\mathrm{R} 10(n=20)$} & \multicolumn{2}{|l|}{$\mathrm{R} 20(n=15)$} & \multicolumn{2}{|c|}{$\mathrm{A} 10(n=16)$} & \multicolumn{2}{|l|}{ A20 $(n=18)$} \\
\hline & baseline & at 6 months & baseline & at 6 months & baseline & at 6 months & baseline & at 6 months & baseline & at 6 months \\
\hline TC & $230.5 \pm 18.7$ & $208.8 \pm 19.3^{* *}$ & $259.8 \pm 33.3$ & $185.0 \pm 33.3^{* *}$ & $302.7 \pm 52.3$ & $214.6 \pm 44.1^{* *}$ & $260.4 \pm 23.2$ & $186.5 \pm 22.7^{* *}$ & $277.4 \pm 42.1$ & $205.3 \pm 27.6^{* *}$ \\
\hline LDL-c & $152.9 \pm 12.6$ & $135.7 \pm 13.4^{* *}$ & $176.6 \pm 25.3$ & $105.5 \pm 25.8^{* *}$ & $214.8 \pm 50.9$ & $134.0 \pm 48.8^{* *}$ & $180.1 \pm 19.3$ & $113.9 \pm 24.7^{* *}$ & $191.2 \pm 31.5$ & $129.1 \pm 27.2^{* *}$ \\
\hline HDL-c & $47.1 \pm 9.4$ & $47.6 \pm 9.1^{*}$ & $49.6 \pm 12.4$ & $53.8 \pm 12.5^{* *}$ & $50.8 \pm 17.2$ & $54.2 \pm 21.0^{* *}$ & $43.4 \pm 5.7$ & $47.9 \pm 7.9^{* *}$ & $45.3 \pm 11.7$ & $47.3 \pm 11.7^{* *}$ \\
\hline TG & $152.5 \pm 65.9$ & $127.7 \pm 52.8^{* *}$ & $167.9 \pm 49.6$ & $128.7 \pm 41.5^{* *}$ & $185.0 \pm 109.6$ & $131.8 \pm 54.4^{* *}$ & $184.2 \pm 80.3$ & $123.1 \pm 35.2^{* *}$ & $204.3 \pm 108.3$ & $144.3 \pm 61.7^{* *}$ \\
\hline TSH & $1.59 \pm 0.91$ & $1.55 \pm 0.74^{*}$ & $1.96 \pm 0.97$ & $1.72 \pm 0.79 *$ & $1.23 \pm 0.82$ & $1.33 \pm 0.73 *$ & $1.96 \pm 0.89$ & $1.98 \pm 0.85^{*}$ & $2.04 \pm 0.92$ & $1.86 \pm 0.95^{*}$ \\
\hline
\end{tabular}

Values are expressed as means \pm SD. R10, rosuvastatin $10 \mathrm{mg}$; R20, rosuvastatin $20 \mathrm{mg}$; A10, atorvastatin $10 \mathrm{mg}$; A20, atorvastatin 20 mg; TC, total cholesterol; LDL-c, low-density lipoprotein cholesterol; HDL-c, high density lipoprotein cholesterol; TG, triglycerides; TSH, thyrotropin-stimulating hormone. ${ }^{*} p<0.05$; ${ }^{* *} p<0.05$.

because of lack of compliance. The control group comprised 37 patients who were not given statins. The study was ultimately completed with 106 patients ( 37 controls and 69 in the statin group). Of these 106, 62 were female (58.5\%) and 44 were male $(41.5 \%)$. Their mean age was $53.22 \pm 10.26$ years (range $25-81$ years). Fortytwo patients (39.6\%) had type 2 diabetes (DM), 49 (46.2\%) had hypertension (HT), and $12(11.3 \%)$ had coronary heart disease. Their body mass index was $28.26 \pm 4.29$ and their rate of smoking was $24.5 \%$. All patients were residing in Ankara, a region with mild-to-moderate iodine deficiency (Table 1) [21].

The $37(34.9 \%)$ patients in the control group were prescribed lifestyle and dietary changes for their hyperlipidemia. Of the 69 patients receiving drug treatment, 20 received rosuvastatin $10 \mathrm{mg}$ (19.8\%), 15 received rosuvastatin $20 \mathrm{mg}$ (14.9\%), 16 received atorvastatin $10 \mathrm{mg}(15.8 \%)$, and 18 received atorvastatin $20 \mathrm{mg}(17.8 \%)$. The mean rosuvastatin and atorvastatin dose was 14.3 and $21.7 \mathrm{mg}$, respectively.

\section{Methods}

Patients assessed as requiring statin treatment were assigned by a simple random sampling method to receive either atorvastatin (10-20 mg daily) or rosuvastatin (10-20 mg daily). The study groups were as follows: group 1 (assessed as not requiring statin treatment), were advised to make therapeutic lifestyle changes $(n=37)$, group 2 received rosuvastatin $(n=35: 20$ received $10 \mathrm{mg}$ [R10] and 15 received $20 \mathrm{mg}$ [R20]), and group 3 received atorvastatin ( $n=34: 16$ received $10 \mathrm{mg}$ [A10] and 18 received $20 \mathrm{mg}$ [A20]). Patients receiving statins were also advised about therapeutic lifestyle changes [20].

The patients in the control group were scheduled for follow-up at 0 and 6 months and those in the statin groups at 0,1 , and 6 months. Serum concentrations of low-density lipoprotein cholesterol (LDL-c), high-density lipoprotein cholesterol (HDL-c), triglycerides (TG), aspartate aminotransferase, alanine aminotransferase, gamma glutamyl transferase, creatine kinase, and thyrotropin-stimulating hormone (TSH) were measured at admission and at 6 months. Concentrations of hepatic enzymes and creatine kinase were measured in the first month of treatment in patients receiving statins. Total cholesterol, HDL-c, and TG concentrations were measured using enzymatic assay (Boehringer, Mannheim, Germany). LDL-c was calculated using the Friedewald formula: LDL-c $=$ total cholesterol $-($ HDL-c + TG/5). Thyroid function was evaluated by measuring the relevant variables by immunochemoluminescence assays with an automated analyzer (Immulite 2000; Diagnostic Products, Los Angeles, CA, USA).

Effects of Statins on the Thyroid Gland
Thyroid ultrasonography was performed on all patients at admission and at 6 months by the same researcher (C.D.) using a 10$\mathrm{MHz}$ linear probe (Logic 5 Pro; GE Medical Systems, Madison, WI, USA). The size of the thyroid gland and each nodule identified was measured in 3 dimensions. The volume of the thyroid gland was calculated by using the following ellipsoid formula:

Volume $(\mathrm{mL})=$ Depth $(\mathrm{cm}) \times$ Width $(\mathrm{cm}) \times$ Length $(\mathrm{cm}) \times$ 0.479 [22].

\section{Statistical Analysis}

Statistical analyses were performed by using SPSS for Windows v21.0. Mean values in independent groups were compared by using an independent-samples $t$ test and means of nonparametric data with the Mann-Whitney $U$ test. Rates of groups were compared by using the $\chi^{2}$ test. Mean values in dependent groups were compared by using a paired-samples $t$ test and means of nonparametric data of dependent groups with the Wilcoxon test. $p<0.05$ was considered statistically significant.

\section{Results}

The statin treatment groups did not differ significantly from each other or the control group with respect to sex, age, weight, BMI, smoking status, or the presence of coronary heart disease. As expected, a statistically significant greater proportion of patients had DM and HT in the drug treatment groups than in the control group.

In addition to statin treatment, the routine use of 3 other groups of drugs for longer than 6 months was also assessed: (a) drugs for DM (oral antidiabetic drugs including metformin, sulfonylurea, glinide, acarbose, and gliptins as well as insulin and combination therapies); (b) drugs for HT (angiotensin-converting enzyme inhibitors, angiotensin receptor blockers, $\beta$ blockers, calcium-channel blockers, and diuretics, or combinations thereof); and (c) acetylsalicylic acid, vitamin D, and omega-3. While all these drugs were used significantly less frequently in the control group than in the drug treatment groups, the drug subgroups did not differ in this respect. 
Table 3. Thyroid volume before and after intervention

\begin{tabular}{llll}
\hline Group & \multicolumn{2}{l}{ Thyroid volume, $\mathrm{mL}$} & \multirow{2}{*}{$p$} \\
\cline { 2 - 3 } & pretreatment & posttreatment & \\
\hline Control $(n=37)$ & $12.4 \pm 4.4$ & $12.4 \pm 4.46$ & 0.97 \\
R10 $(n=20)$ & $13.2 \pm 8.7$ & $13.1 \pm 8.5$ & 0.16 \\
R20 $(n=15)$ & $12.0 \pm 4.3$ & $11.5 \pm 4.0$ & $0.03^{*}$ \\
A10 $(n=16)$ & $11.7 \pm 4.1$ & $11.5 \pm 4.2$ & 0.18 \\
A20 $(n=18)$ & $12.4 \pm 3.4$ & $12.2 \pm 3.3$ & 0.07 \\
\hline
\end{tabular}

Values are expressed as means $\pm \mathrm{SD} . \mathrm{R} 10$, rosuvastatin $10 \mathrm{mg}$; $\mathrm{R} 20$, rosuvastatin $20 \mathrm{mg}$; A10, atorvastatin $10 \mathrm{mg}$; A20, atorvastatin 20 mg. $* p<0.05$.

Table 4. Mean maximum nodule diameter before and after intervention

\begin{tabular}{lrrl}
\hline Group & \multicolumn{2}{l}{$\begin{array}{l}\text { Maximum nodule diameter, } \\
\text { mm }\end{array}$} & \\
\cline { 2 - 3 } & pretreatment & posttreatment & \\
\hline Control $(n=11)$ & $6.62 \pm 2.38$ & $6.72 \pm 2.73$ & 0.579 \\
R10 $(n=7)$ & $16.14 \pm 9.52$ & $15.42 \pm 9.10$ & $0.047^{*}$ \\
R20 $(n=4)$ & $9.50 \pm 4.65$ & $8.92 \pm 4.67$ & 0.331 \\
A10 $(n=9)$ & $6.88 \pm 2.47$ & $6.66 \pm 2.39$ & 0.447 \\
A20 $(n=12)$ & $9.75 \pm 6.60$ & $9.53 \pm 6.59$ & 0.229 \\
\hline
\end{tabular}

Values are expressed as means $\pm \mathrm{SD}$. R10, rosuvastatin $10 \mathrm{mg}$; $\mathrm{R} 20$, rosuvastatin $20 \mathrm{mg}$; A 10, atorvastatin $10 \mathrm{mg}$; A20, atorvastatin 20 mg. $* p<0.05$.

Following treatment, there were statistically significant decreases from the levels at baseline of total cholesterol, LDL-c, and TG, in both the control and treatment groups $(p<0.01)$. An apparent increase from baseline levels in HDL concentrations was not statistically significant in the control group at the end of the study; however, these increases were statistically significant in the drug treatment groups $(p<0.05)$. No statistically significant changes were observed between pre- and posttreatment TSH concentrations in any group (Table 2).

Upon entry to the study, the mean total thyroid volume of all 106 patients was $12.46 \pm 5.21 \mathrm{~mL}$, and at 6 months it was $12.39 \pm 5.11 \mathrm{~mL}(p>0.05)$, a nonsignificant change. Although there were no statistically significant changes in total thyroid volume at 6 months in groups 1, 2 and 3, a significant reduction was seen in the R20 subgroup of group 2 after treatment $(p=0.034$; Table 3$)$.

Nodules were detected in 43 (40.56\%) patients; 21 had single nodules and 22 had multiple nodules. The mean maximum nodule diameter upon entry was $6.62 \pm 2.38$ $\mathrm{mm}$ in the control group and $10.31 \pm 2.93 \mathrm{~mm}$ in the drug treatment groups. There was no change in the number of nodules at 6 months.

The posttreatment maximum nodule diameter was $6.72 \pm 2.73 \mathrm{~mm}$ (a mean increase of $0.10 \mathrm{~mm}$ ) in the control group and $9.98 \pm 6.76$ (a mean decrease of $0.33 \mathrm{~mm}$ ) in the drug group; these changes were not statistically significant. However, compared with the values at admission, a statistically significant decrease in maximum nodule diameter was observed in the R10 group $(n=18)$ at 6 months. Seven of these 18 patients had nodules with a mean diameter of $16.14 \pm 9.5 \mathrm{~mm}$ at admission. Although the number of nodules did not change after treatment, the mean nodule diameter decreased to $15.42 \pm 9.1 \mathrm{~mm}(p=$ 0.047; Table 4).

\section{Discussion}

This study found no statistically significant differences in TSH concentrations, thyroid volume, the number of nodules, or maximum nodule diameters between patients who did and did not receive statin treatment.

At six months of treatment, there was no difference in the patients receiving the 2 types of statins. However, a comparison of the subgroups with the corresponding values in the control group revealed that total thyroid volume was significantly smaller in the R20 group $(p<0.05)$, and maximum nodule diameter significantly smaller in the R10 group $(p<0.05)$. The lack of reduction in the maximum nodule diameter in the R20 group could have been due to the smaller number of patients with nodules in this group (R10 $n=7$ vs. R20 $n=4$ ) or to the relatively short duration of the study or both. The effects on thyroid volume after 6 months of treatment with $20 \mathrm{mg}$ rosuvastatin are in agreement with data obtained from retrospective patient surveys and in vitro and in vivo animal studies, and they may be related to the antiproliferative and apoptotic effects of prenylation inhibition on the mevalonate pathway $[11,12,16,17]$. The number of nodules did not change with treatment.

First discovered in 1976 and in continuous use since 1981, statins have pleiotropic effects that have been known and studied extensively for 15 years [23]. Most of these effects are associated with the inhibition of the mevalonate pathway $[3,4]$.

Vitale et al. [12] showed in vitro that statins induce apoptosis by multiplying thyroid cells independently of p53 through protein synthesis and a mechanism based on
Demir/Anil/Bozkus/Mousa/Kut/Nar/ Tutuncu 
caspase enzymes. Apoptosis, a process of the self-destruction of cells, may be physiological or pathological. An apoptotic pathway may be initiated by various cytokines, growth factors, hormone deficiencies, or drugs, via triggering the apoptotic system threshold. Various genes are associated with the regulation of apoptosis. The p53 gene controls the RNA transcription of pro- and antiapoptotic genes and plays a key role in cell death $[13,24]$.

Lovastatin, an HMG-CoA reductase inhibitor, has been shown to induce apoptosis in malignant glial tumors and prostate stromal tumors $[25,26]$. Lovastatin inhibits the reduction of HMG-CoA reductase to mevalonate, the precursor of isopentenyl pyrophosphate. It also prevents the formation of mevalonate, geranyl pyrophosphate, farnesyl pyrophosphate, and all-trans-geranylgeranyl pyrophosphate, thus preventing these molecules from proliferating with farnesyl and geranylgeranyl transferase, and also preventing the transfer of the Ras super-family (Rho, Raf, Rac, and Rap), which plays a role in major cellular functions like cell adhesion or motility, to small GTP-binding proteins. It was observed that in cases of prenyl modification deficiency, small GTP-binding proteins fail to form a complex with target proteins, and this disruption in their functions induces apoptosis.

To investigate the apoptotic effects of HMG-CoA reductase inhibition on thyroid cell cultures, Vitale et al. [12] studied the effects of lovastatin on normal and neoplastic thyroid cells, and observed cellular rounding in 24 $\mathrm{h}$ and DNA fragmentation and apoptosis in $48 \mathrm{~h}$, in both normal and neoplastic cadaver thyroid cell series. When mevalonate and the protein synthesis inhibitor, cycloheximide, were added, all effects of lovastatin were blocked in a dose-dependent manner. Their finding that lovastatin is a potent inducer of apoptosis in multiplying thyroid cell cultures supports the contention that prenylation inhibitors may have therapeutic potential, not only in prostate cancers and prostatic hyperplasia, but also in the proliferative processes of the thyroid.

In another study, rat thyroid cells with Ras-transformation were found to be more sensitive to HMG-CoA reductase inhibitors than normal cells. Ras mutation activation and other oncogenes are common in thyroid tumors, and ras mutations may also be present in the nodular hyperplasia of the thyroid. This suggests that statins have a potential use for treatment of benign and malignant diseases of the thyroid $[12,14,24]$.

Zhong et al. [24] studied the effects of lovastatin on apoptosis and the induction of differentiation in anaplastic thyroid carcinoma (ATC) cells refractory to conventional treatments. As a result, a high dose $(50 \mu \mathrm{M})$ of

Effects of Statins on the Thyroid Gland lovastatin was found to induce apoptosis, while smaller doses $(25 \mu \mathrm{M})$ were seen to trigger cytomorphological differentiation. An increase in the expression of thyroglobulin was also detected in the ATC cells. These results supported the use of HMG-CoA reductase inhibitors for redifferentiation in the treatment of ATC. In a more recent study [27], this group demonstrated that lovastatin at lower concentrations exerted an antiproliferation effect on human ATC cells through increases of p27 mediated by the reduction of Rho geranylgeranylation. These results suggested that lovastatin increases the levels of p27 protein through actions on RhoA/ROCK signaling and ubiquitination pathways, and inhibited proliferation of ARO cells by increasing the level of $\mathrm{p} 27$ protein. They concluded that lovastatin exerts both antiproliferative and differentiation-inducing effects on human ATC cells, probably rendering them more sensitive to radioactive iodine therapy.

A preclinical study indicated that epidermal growth factor (EGF) is involved in the proliferation and migration of differentiated thyroid cancer [28]. Overexpression of EGF or EGF receptor (EGFR) was observed in most of the thyroid cancer cells including the ATC cells, and was associated with a poor prognosis in patients with metastatic thyroid cancer. EGFR was therefore identified as a novel therapeutic target for treating patients with ATC $[29,30]$.

Several studies have demonstrated that lovastatin and simvastatin can inhibit cell adhesion, migration, and invasion $[31,32]$. Recently, it has been shown that EGF can induce Cyr61 expression through the activation of the EGFR/ERK/CREB signaling pathway, and that EGF-induced Cyr61 is involved in the regulation of cell migration in ATC cells [33]. Combined treatment with lovastatin and troglitazone markedly inhibited cell migration by inhibiting EGF-induced Cyr61 expression in ATC cells. The authors reported that these results may provide a useful future strategy in the treatment of ATC [34].

In a 2006 study conducted on rats, Laezza et al. [15] reported that HMG-CoA reductase inhibitors inhibited propylthiouracyl-induced goiter by $\mathrm{p} 21$ ras-MAPK pathway modulation. Even though different molecular and genetic processes have been proposed, results show that lovastatin is an effective inhibitor in goitrogenesis with p21 ras farnesylation inhibition. The authors concluded that mevalonate pathway inhibition may create antiproliferative effects in both benign and malignant thyroid tissue.

The first clinical study on thyroid volume and nodule prevalence in dyslipidemia patients on statins was con- 
ducted in 2008 by Cappelli et al. [16]. They retrospectively assessed 137 patients who had been using statins for at least 5 years, and found a reduction in thyroid volume, and the prevalence, number, and volume of nodules with HMG-CoA reductase inhibitor treatment. Nodule prevalence among patients who had been receiving various statin treatments in the previous 5 years was $36.3 \%$ whereas the rate in a nontreatment control group was $67.9 \%$ $(p<0.001)$. Thyroid and nodule volumes were also significantly smaller in the group that had received statins for a long time.

In another retrospective study on $70 \mathrm{DM}$ subjects who were on statins for long periods and lived in a region with iodine deficiency, no difference in thyroid size, or nodule prevalence, number, and volume was found between the drug and control groups $(n=98)$. However, when patients were grouped into age brackets, thyroid nodules were less prevalent in patients aged $60-65$ years $(n=12)$ than in control patients in the same age bracket $(n=27)$ (33 vs. $70 \%, p=0.04$ ). These findings are thought to be associated with the possible antiproliferative effects of statins, an association believed to be weak in regions with iodine deficiency [17].

In vitro and in vivo studies on breast cancer and melanoma suggest that only hydrophobic statins have useful pleiotropic effects [35, 36]. However, Cappelli et al. [16], in a retrospective study on 137 patients on statins (104 on simvastatin [hydrophobic], 29 on atorvastatin [hydrophilic], 1 on pravastatin, and 1 on rosuvastatin), did not detect a relationship between the number and volume of thyroid nodules and molecule type. While the duration of statin use is reportedly a stronger predictor than the daily dose for a reduction in the risk of developing prostate cancer, Shannon et al. [37] concluded that nodules are less prevalent among patients taking a greater mean daily dose, independent of duration.

In our study, hydrophilic rosuvastatin $(20 \mathrm{mg})$ had a significant effect on total thyroid volume. The maximum reduction in nodule diameter was observed in the R10 group. The lack of a reduction in maximum nodule diameter in patients in the R20 group may be attributable either to the small number of patients with nodules $(n=4)$ in this group, or to the absence of a relationship between the pleiotropic effects of statins on the thyroid gland and the dosage.

Very few published studies have focused on the relationship between the pleiotropic effects of statins and common thyroid diseases. Existing studies are in vitro studies, animal studies, case observations, or retrospective studies. We obtained results in 2 of our study groups that suggest that the antiproliferative effects of statins can result in smaller thyroid volumes and nodule diameters. Statins may thus have beneficial therapeutic effects in thyroid diseases. Our study was limited by its short duration and small case numbers.

Future monitoring studies with larger groups of patients and of longer duration are needed to fully understand the molecular mechanisms of the action of prenylation inhibitors on antiproliferative and apoptotic pathways, and to determine the appropriate and usable molecules and daily dosages. Our findings support the possible use of HMG-CoA reductase inhibitors in the treatment of benign and malignant diseases of the thyroid.

\section{Conclusion}

We compared the effects of 2 statins on thyroid function, volume, and nodule prevalence in a prospective, controlled setting. Posttreatment, we found a significant decrease in total thyroid volume in the R20 group and maximum nodule diameter in the $\mathrm{R} 10$ group compared with the changes in the control group. Rosuvastatin may exert antiproliferative effects on cells by inhibiting the mevalonate synthesis pathway and decreasing isoprenoid compound formation.

\section{Acknowledgement}

This study was supported by the Research Fund of the Baskent University (project No. KA12/02).

\section{Disclosure Statement}

The authors declare no conflicts of interest.

$\begin{aligned} \text { References } & \begin{array}{l}\text { Takemoto M, Liao JK: Pleiotropic effects of } \\ \text { 3-hydroxy-3-methylglutaryl coenzyme A red } \\ \text { inhibitors. Arterioscler Thromb Vasc Biol } \\ \\ 2001 ; 21: 1712-1719 .\end{array} \\ 2 & \text { Liao JK, Laufs U: Pleiotropic effects of statins. } \\ & \text { Annu Rev Pharmacol Toxicol 2005;45:89- } \\ & 118 . \\ 3 & \text { Vaughan CJ, Murphy MB, Buckley BM: } \\ & \text { Statins do more than just lower cholesterol. } \\ & \text { Lancet 1996;348:1079-1082. } \\ & \text { Buhaescu I, Izzedine H: Mevalonate pathway: } \\ & \text { a review of clinical and therapeutical implica- } \\ & \text { tions. Clin Biochem 2007;40:575-584. }\end{aligned}$

Demir/Anil/Bozkus/Mousa/Kut/Nar/ Tutuncu 
5 Goldstein JL, Brown MS: Regulation of the mevalonate pathway. Nature 1990;343:425430.

-6 Bonetti PO, Lerman LO, Napoli C, et al: Statin effects beyond lipid lowering - are they clinically relevant? Eur Heart J 2003;24:225-248.

7 Dulak J, Józkowicz A: Anti-angiogenic and anti-inflammatory effects of statins: relevance to anti-cancer therapy. Curr Cancer Drug Targets 2005;5:579-594.

-8 Vallianou NG, Kostantinou A, Kougias M, et al: Statins and cancer. Anticancer Agents Med Chem 2014;14:706-712.

-9 Alexandre L, Clark AB, Bhutta HY, et al: Statin use is associated with reduced risk of histologic subtypes of esophageal cancer: a nested case-control analysis. Gastroenterology 2014;146:661-668.

10 Farina HG, Bublik D, Alonso DF, et al: Lovastatin alters cytoskeleton and inhibits experimental metastasis of mammary carcinoma cells. Clin Exp Metastasis 2002;19:551-559.

11 Bifulco M: Therapeutic potential of statins in thyroid proliferative disease. Nat Clin Pract Endocrinol Metab 2008;4:242-243.

12 Vitale M, Di Matola T, Rossi G, et al: Prenyltransferase inhibitors induce apoptosis in proliferating thyroid cells through a p53-independent, CrmA-sensitive, and caspase-3like protease-dependent mechanism. Endocrinology 1999;140:698-704.

-13 Demierre MF, Higgins PD, Gruber SB, et al: Statins and cancer prevention. Nat Rev Cancer 2005;5:930-945.

-14 Hofman A, John P, Schaffarich MP, et al: Statins as a new therapeutic approach in dedifferentiated thyroid cancer? Nuklear Medizin 2006;45:28-30.

-15 Laezza C, Mazziotti G, Fiorentino L, et al: HMG-CoA reductase inhibitors inhibit rat propylthiouracil-induced goiter by modulating the ras-MAPK pathway. J Mol Med 2006; 84:967-973.

16 Cappelli C, Castellano M, Pirola I, et al: Reduced thyroid volume and nodularity in dyslipidaemic patients on statin treatment. Clin Endocrinol 2008;68:16-21.
17 Chon MG, Suk JH, Oh KH, et al: Influence of long-term statin use in type 2 diabetic patients on thyroid nodularity in iodine-sufficient area. Exp Clin Endocrinol Diabetes 2011;119: 497-501.

18 Aghini-Lombardi F, Antonageli L, Martino E, et al: The spectrum of thyroid disorders in an iodine-deficient community: the Pescopagano survey. J Clin Endocrinol Metab 1999;84: 561-566.

19 Andersen-Ranberg K, Jetine B, Hoier-Madsen $M$, et al: Thyroid function, morphology and prevalence of thyroid disease in a population based study of Danish centenarians. J Am Geriatr Soc 1998;47:1238-1243.

20 Executive summary of the third report of the National Cholesterol Education Program (NCEP) Expert Panel on Detection, Evaluation, and Treatment of High Blood Cholesterol in Adults (Adult Treatment Panel III). JAMA 2001;285:2486-2497.

-21 Erdoğan MF, Ağbaht K, Altınsu T, et al: Current iodine status in Turkey. J Endocrinol Invest 2009;32:617-622.

22 Brunn J, Block U, Ruf G, et al: Volumetric analysis of thyroid lobes by real-time ultrasound. Dtsch Med Wochenschr 1981;106: 1338-1340.

23 Endo A, Kuroda M, Tanzawa K: Competitive inhibition of 3-hydroxy-3-methylglutaryl coenzyme A reductase by ML-236A and ML236B fungal metabolites, having hypocholesterolemic activity. FEBS Lett 1976;72:323326.

24 Zhong W, Wang C, Chang T, Lee W: Lovastatin induces apoptosis of anaplastic thyroid cancer cells via inhibitor of protein geranylgeranylation and de novo protein synthesis. Endocrinology 2003;144:3852-3859.

25 Jones KD, Couldwell WT, Hinton DR, et al: Lovastatin induces growth inhibition and apoptosis in human malignant glioma cells. Biochem Biophy Res Commun 1994;205: 1681-1687.

26 Padayatty SJ, Marcelli M, Shao TC, et al: Lovastatin induced apoptosis in prostate stromal cells. J Clin Endocrinol Metab 1997;82:14341439.

27 Zhong WB, Hsu SP, Ho PY, et al: Lovastatin inhibits proliferation of anaplastic thyroid cancer cells regulation of p27 by interfering with the Rho/ROCK-mediated pathway. Biochem Pharmacol 2011;82:1663-1672.
28 Hoelting T, Siperstein AE, Clark OH, et al: Epidermal growth factor enhances proliferation, migration, and invasion of follicular and papillary thyroid cancer in vitro and in vivo. Clin Endocrinol Metab 1994;79:401-408.

29 van der Laan BF, Freeman JL, Asa SL: Expression of growth factors and growth factor receptors in normal and tumorous human thyroid tissues. Thyroid 1995;5:67-73.

-30 Ensinger C, Spizzo G, Moser P, et al: Epidermal growth factor receptor as a novel therapeutic target in anaplastic thyroid carcinomas. Ann NY Acad Sci 2004;1030:69-77.

31 Zhong WB, Liang YC, Wang CY, et al: Lovastatin suppresses invasiveness of anaplastic thyroid cancer cells by inhibiting Rho geranylgeranylation and RhoA/ROCK signaling. Endocr Relat Cancer 2005;12:615-629.

32 Xiao Y, Liang L, Pan Y, et al: Inhibitory effects of simvastatin on migration and invasion of rheumatoid fibroblast-like synoviocytes by preventing geranylgeranylation of RhoA. Rheumatol Int 2013;33:389-399.

33 Chin LH, Hsu SP, Zhong WB, et al: Involvement of cysteine-rich protein 61 in the epidermal growth factor-induced migration of human anaplastic thyroid cancer cells. Mol Carcinog 2016;55:622-632.

34 Chin LH, Hsu SP, Zhong WB, et al: Combined treatment with troglitazone and lovastatin inhibited epidermal growth factor-induced $\mathrm{mi}$ gration through the downregulation of cysteine-rich protein 61 in human anaplastic thyroid cancer cells. PLoS One 2015; 10:e0118.

35 Farina HG, Bublik DR, Alonso DF, et al: Lovastatin alters cytoskeleton and inhibits experimental metastasis of mammary carcinoma cells. Clin Exp Metastasis 2002;19:551-559.

36 He L, Mo H, Hadisusilo S, et al: Isoprenoids suppress the growth of murine B16 melanomas in vitro and in vivo. J Nutr 1997; 127:668674 .

37 Shannon J, Tewoderos S, Garzotto M, et al: Statins and prostate cancer risk: a case-control study. Am J Epidemiol 2005;162:318325 . 